\title{
हिन्दुस्तानी शास्त्रीय गायन में अंलकारों का महत्व
}

श्वेता काले ${ }^{1}$

संगीत मानव जाति की एक विशिष्ट उपलब्धि है। यह एक ऐसा ईश्वरीय वरदान है जो उसे दिव्य लोक की अनुभूति कराता है। यह मानव वृत्ति से जुड़ा हुआ मानवीय संस्कृति का परिचायक ही नहीं अपितु जन्म से लेकर मृत्यु तक अनवरत रूप से मानव शरीर की चेतना के साथ सम्बद्ध रहता है। यह एक सजीव परन्तु अमूर्त कला है जिसमें कलाकार एवं श्रोता दोनों परमानंद की अनुभूति करते हैं।

\section{शास्त्रीय संगीत}

शास्त्रीय संगीत से अभिप्राय उस संगीत से हैं जो परम्परानुसार एवं शास्त्रबद्ध होता हैं। इसमें कई बातों का ध्यान रखा जाता है -

1. कण्ठ साधना एवं आवाज की पूर्ण तैयारी।

2. स्वरों का शुद्ध एवम् उचित लगाव।

3. स्वर-लगाव द्वारा अनुपम सौदर्य का निर्माण।

4. राग प्रस्तुतिकरण में राग नियमों का ध्यान रखना।

5. राग व ताल पर पूर्ण अधिकार व इनका सटीक प्रयोग।

6. राग प्रस्तुतिकरण की विभिन्न शैलियों का प्रयोग।

जब किसी नयी शेली का जन्म होता है तो वह एक ही पीढ़ी में शास्त्रीयता की मार्यदा नहीं पाती अपितु कम से कम तीन पीढ़ियों तक निरंतर प्रवाह के पश्चात् ही वह शैली मान्यता प्राप्त करती है। उदाहरण के लिए 18 वीं शताब्दी के पूर्वार्द्ध तक 'ख्याल' को शास्त्रीय मान्यता प्राप्त नहीं थी और अदारंग सदारंग जैसे गायकों ने भी इसे अपने पुत्रों को नहीं सिखाया क्योंकि यह 'जनाना' गाना माना जाता था। किन्तु उनके शिष्यों के अथक प्रयासों से ही इसे शास्त्रीय संगीत की मर्यादा प्राप्त हुई। वर्तमान समय ख्याल शास्त्रीय संगीत का पर्यावाची बन गया है।

हिन्दुस्तानी शास्त्रीय संगीत पर दृष्टिपात करें तो यह वैदिक युग से लेकर आधुनिक काल तक के अनके परिवर्तनों सहित पल्लवित होता रहा है। संगीत, विशेषतः शास्त्रीय संगीत, युगों की चेतना के परिणाम स्वरूप फलता-फूलता और विकसित होता रहा है। शास्त्रीय संगीत का आधार नाद है जो आकाश का गुण होने से सर्वत्र व्यापक है। नाद के संयोग से ही वर्णों का उच्चारण होता है। वर्ण से शब्द बनते हैं। शब्दों से भाषा तथा भाषा से ही संसार के कार्य चलते हैं। अतः नाद महत्वपूर्ण एवं मूलभूत तत्व है। नाद और श्रुति से स्वरों का निर्माण होता है। भले ही शास्त्रकारों न 22 श्रुतियों की बात की है परन्तु विद्वजन अभी तक श्रुतियों की संख्या तथा आकार के सम्बन्ध में एकमत नहीं हो पाये हैं। यह कहना बिल्कुल उचित है कि भारतीय संगीत स्वर एवं श्रुति प्रधान न होकर नाद प्रधान है। जो नाद दीर्घ रूप धारण कर लेता है वह स्वर बन जाता है।

नाद, श्रुति एवं स्वर एक दूसरे के पूरक हैं। स्वर की स्थापना तथा साधना ही भारतीय संगीत में सर्वोपरि है। स्वरों को विभिन्न क्रमों में ढ़ालकर अलंकारों का निर्माण होता है। जिस प्रकार मानवीय शरीर की रंचना पंच मूलभूत तत्वों, पृथ्वी, जल, अग्नि, आकाश, वायु से मानी गई है, उसी प्रकार अलंकारों में नाद, श्रुति, स्वर वर्ण एवं निरंतर क्रम में तत्व समाहित रहते हैं। अलंकारों का उच्चारण और स्वर साधना द्वारा ही स्वरों के शुद्ध लगाव को साधा जा सकता है जो श्रुतियों के ज्ञान द्वारा ही सम्भव है। श्रुति नाद का परिष्कृत रूप है। इस प्रकार नाद, श्रुति एवं स्वर अलंकारों के अभ्यास द्वारा साध्य हो सकते हैं।

अलंकार शब्द का शाब्दिक अर्थ है आभूषण, गहना अर्थात् किसी व्यक्ति या वस्तु को सुन्दर बनाने वाले तत्व। अलंकार दो शब्दों के मेल से बना हैं:- अलम्+करोतिः। अलम् का अर्थ है पूर्ण और करोति का अर्थ करना, यानि जो तत्व किसी वस्तु को पूर्ण

1 शोध-छात्रा, दिल्ली। 
करता है। भारतीय संगीत में अलंकार शब्द भी संगीत को अलंकृत करने के तात्पर्य से प्रयुक्त हुआ है। गायन के सौंदर्यवृद्धि ही अलंकारों से होती है। पं。 ओंकारनाथ ठाकुर जी के कथन में वर्णों के अनुसार जब किसी निश्चित क्रम को अपनाकर कोई स्वर रचना बनाई जाती है तो उसे अलंकार कहते हैं। पं० भातखण्ड़े 'क्रमिक पुस्तक मालिका' में विद्वानों द्वारा दी गई अलंकार शब्द की व्याख्या इस प्रकार है-

\section{विशिष्टवर्णसंदर्भमलकांर प्रचक्षते}

अर्थात् कुछ नियमित वर्णों के समुदाय को अलंकार कहते हैं। विशिष्ट स्वर समूह ही अलंकार बन जाते हैं। उन्हें भी आरोही-अवरोहादि वर्णों की आवश्यकता होती है। प्रचार में गुणीजन अलंकारों को पलटे भी कहते हैं।

प्राचीन ग्रन्थों में स्वरों के विभिन्न समूह बनाने की एक पद्धति, खंडमेरू, का विवरण भी प्राप्त होता है। मतंग ने 'बृहदेशी' स्वर-प्रस्तार का वर्णन किया है। एक मूर्छना का स्वर सप्तक लेकर उसका प्रस्तार करने से अर्थात् स्वरों को अलग-अलग क्रम में रखने से 5040 प्रकार के तान प्रस्तार मिलते है। तानों की उपरोक्त प्रस्तार की विशेष पद्धति को खंडमेरू का नाम दिया गया है।

कंट-साधना एवं स्वराभ्यास का गायन संगीत में महत्वपूर्ण स्थान है। जहां एक ओर कंट-साधना द्वारा आवाज की गुणवत्ता को निखारा जा सकता है वहीं दूसरी ओर स्वराभ्यास द्वारा गले की कसरत या उसके घुमाव-फिराव पर अधिक ज़ोर रहता है। यदि कंठ सुयोग्य होगा तो ही संगीतज्ञ अपने मन के भावों को भली प्रकार अभिव्यक्त कर सकता है।

किराना घराने की प्रतिष्ठित कलाकार डा॰ प्रभा अत्रे जी इसके विषय में कहती हैं-

"सरगम (बारह स्वरों) की साधना तो आजीवन करनी होती है किंतु कंट-साधना के लिए यह भी देखना होता है कि गले का नैसर्गिक गुण-धर्म किस प्रकार का है और क्षमता कितनी है। वस्तुतः आवाज में विविधता एवं फिरत की क्षमता का होना अति आवश्यक है।"

संगीत अभ्यास के लिए अलंकारो का विधिवत अभ्यास अत्यंत आवश्यक है और यही संगीत का मूल मंत्र है। इससे स्वर लगाने का सही अंदाज मिलता है। कठिन व जटिल तानों के निर्माण में इससे बहुत सहायता मिलती है। टप्पा जैसे कठिन गायकी में जिसमें एक विशेष प्रकार की तानों का प्रयोग किया जाता है, अलंकारों का अभ्यास बहुत अधिक काम आता है। इसीलिये अलंकारों का विधिवत अभ्यास करना अति आवश्यक है। अलंकारों के निरंतर अभ्यास से कंट व स्वरों की तैयारी शीघ्रता से होती है व समय बर्बाद नहीं होता है, यदि यह अभ्यास किसी योग्य गुरू के निर्देशन में किया जाए।

संगीत शिक्षण में भी अलंकारों का बहुत अधिक महत्व है। जब भी शिक्षक विद्यार्थी को संगीत की शिक्षा प्रारम्भ करवाता है तो सर्वप्रथम स्वर-लगाव के पश्चात् अलंकारों की शिक्षा देता है जिसके अन्तर्गत वह विद्यार्थी को सात स्वरों के भिन्न-भिन्न स्वर समुदाय बनाकर उनका चमत्कारिक प्रयोग दिखाता है। अलंकारों के प्रयोग से विद्यार्थी के कंठ की तैयारी के साथ-साथ उसकी कल्पनाशक्ति का भी विकास होता है। अतः अलंकारों के द्वारा ही विद्यार्थी में संगीत की नींव डाली जाती है जो उसकी सम्पूर्ण संगीत यात्रा में सौन्दर्यवृद्धि करती है।

राग के विस्तार में भी अलंकार महत्वपूर्ण भूमिका निभाते हैं। जब कोई अलंकार, किसी राग के नियमों में बंधकर प्रयोग में लाया जाता है तब वह तान कहलाता है। ऐसा भी नहीं हैं कि एक तान में एक ही प्रकार के अलंकार का प्रयोग होता हैं, अधिकतर एक तान में अनेक प्रकार के अलंकारों की झलक दिखाई पड़ती है। अलंकार के व्यापक क्षेत्र, महत्व एवं उसके कार्य को देखते हुए यदि अलंकार जैसी चीज को राग-प्रस्तुतिकरण में समाविष्ट न करते हुए सिर्फ रियाज तक सीमित रखेगें तो यह अलकारों के प्रति अन्याय होगा। अलंकार जैसे क्रियात्मक तत्व का संतुलित एवं समझदारी पूर्ण प्रयोग कलाकरों के गायन में कितना महत्वपूर्ण सिद्ध हुआ है, यह बात प्रत्यक्ष है व किसी प्रमाण की मोहताज नहीं है। प्रत्येक घराने की अपनी एक अलग पहचान होती है। इस अलग पहचान के पीछे एक घराने की विशेष शिक्षण पद्धति एवं विशिष्ट अभ्यास-तकनीकों का हाथ रहता है। हर घराने में कंठ साधना से लेकर स्वर साधना, रागाभ्यास व मंच-प्रदर्शन तक के शिक्षण में काफी भिन्नताएं होती हैं जो आगे चलकर घराने की एक अलग गायन शैली को जन्म देती है। 
किराना घराने की गायकी में आलापचारी पर अधिक ध्यान दिया जाता है। जयपुर घराने के गायक राग में अपनी उपज में एक विचित्रता बनाये रखते हैं तथा गायन के लिए वह अप्रचलित रागों के चुनाव पर अधिक बल देते हैं। संगीत घराने का अर्थ एक विशेष प्रकार की गायकी से है जो किसी गुरू विशेष से मौखिक रूप में पीढ़ी-दर-पीढ़ी चलती है। पुराने समय में गुरूजन स्वर-साधना को विशेष स्थान देते थे जिससे राग विस्तार के लिए आलाप से तान तक की सम्पूर्ण तैयारी हो जाती थी। गायकी की विशेषताओं के अनुसार की अलंकारों का अभ्यास करवाया जाता था। अतः किराना घराने की बढ़त, जयपुर घराने की पेचीदा तानें, भिंड़ी बाज़ार घराने की सरगम, दिल्ली व पटियाला घराने की तेज़-तरार गायकी इन सब के पीछे विशिष्ट अलंकारों की भूमिका रहती है।

आज घरानों की गायकी का परिपालन उस प्रकार से नहीं हो रहा तथा आज व्यक्तिवाद की प्रधानता है। गायक कलाकार कहीं से जो भी अच्छा मिले उसे अपनाना चाहते हैं। किसी विशेषता को अपने घराने की न मानकर अस्वीकृत करने का कम प्रचलन है। परन्तु इस परिस्थिति ने भी अलंकारों के महत्व को कम नहीं किया है। गायन में किसी भी प्रकार की विशिष्टता एवं सौंदर्य को अर्जित करने के लिए अलंकारों का अभ्यास आवश्यक है।

सारांश में अलंकारों के मुख्य उद्देश्य निम्नलिखित हैं -
1. आदर्श कंठ का निर्माण।
2. स्वरों की सही लगाव।
3. आवाज का सही प्रयोग।
4. विभिन्न क्रमों में स्वरों का अभ्यास।
5. स्वर-माधुर्य एवं सौंदर्यवृद्धि।
6. राग विस्तार में सहायता।

7. सम्पूर्ण सांगीतिक सौंदर्य की वृद्धि।

वर्तमान समय में शास्त्रीय संगीत के बाह्य सवरूप में समय-समय पर परिवर्तन आते रहे और आज भी यह परिवर्तशील है। कला कभी भी स्थिर नहीं रहती परन्तु यह भी सत्य है कि शास्त्रीय संगीत के मूलभूत नियम व आंतरिक स्वरूप सदैव कायम रहते हैं। अतः अलंकारों की सार्थकता आज भी उतनी ही है जितनी कल थी। आधुनिक परिवेश में जीवन की गति बहुत तेज हो गई है। संगीत जैसी साधनामयी कला के लिए गुरू व शिष्य, अध्यापक व विद्यार्थी सबके पास समय का अभाव है। पहले सालों तक अलंकारों का अभ्यास ही विद्यार्थी को करवाया जाता था ताकि उसका कंठ दोषमुक्त हो जाये एवं कल्पनाशक्ति भी परिपक्व हो जाए उस तरह का प्रयोग आज के शिक्षण में दिखाई नहीं देता। विशेषतः जो विद्यार्थी महाविद्यालय तथा विश्वविद्यालय स्तर पर संगीत को एक विषय के रूप में चुनते हैं वह इस अनुभव से वंचित रह जाते हैं। संगीत कला की गुणवत्ता की दृष्टि से यह रिथति ठीक नहीं है। भारतीय संगीत के सब शास्त्राकार, विद्वान, साधक, गुरूजन तथा चिंतक इस तथ्य को स्वीकार करते हैं कि अलंकारों का भारतीय संगीत में महत्वपूर्ण स्थान है। यदि हमें इस महान धरोहर की रक्षा करनी तन्मयता से करनी है तो अलंकारों को उनका यथायोग्य सथान देना व सही ढंग से उनका प्रयोग करना होगा। राग का अस्तित्व अलंकारों के साथ जुड़ा है। जब तक यह सृष्टि है, संगीत हैं, राग हैं, तब तक अलंकारों की सार्थकता अटल है।

\section{संदर्भ ग्रंथ सूची}

1. चक्रवर्ती इन्द्राणी, संगीत मंजुषा, मित्तल पब्लिकेशन दिल्ली,1988।

2. ठाकुर ओंकारनाथ, संगीतांजली-भाग 4, संगीत कार्यालय हाथरस ।

3. मतंगमुनि, बृहदेशी, संगीत कार्यालय हाथरस प्रथम संस्करण, 1976।

4. विष्णु नारायण भातखण्ड़े, भातखण्ड़े संगीत शास्त्र, भाग-1 अनुवादक-विश्वरम्भरनाथ भट्ट।

5. लक्ष्मी नारायण गर्ग, संगीत अभ्यास अंक-जनवरी, फरवरी1984, संगीत कार्यालय हाथरस।

6. शबनम, भारतीय संगीत में अलंकार, संजय प्रकाशन दिल्ली, प्रथम संस्करण, 2000 । 